Supporting Information for:

\title{
Strain-induced anion-site occupancy in perovskite
}

\section{oxyfluoride films}

Jiayi Wang ${ }^{1}$, Yongjin Shin², Jay R. Paudel ${ }^{3}$, Joseph D. Grassi ${ }^{3}$, Raj K. Sah ${ }^{3}$, Weibing Yang ${ }^{3}$, Evguenia Karapetrova ${ }^{4}$, Abdulhadi Zaidan ${ }^{5}$, Vladimir N. Strocov ${ }^{5}$, Christoph Klewe ${ }^{6}$, Padraic Shafer $^{6}$, Alexander X. Gray ${ }^{3,7}$, James M. Rondinelli², Steven J. May ${ }^{1}$

${ }^{1}$ Department of Materials Science and Engineering, Drexel University, Philadelphia, Pennsylvania 19104, USA

${ }^{2}$ Department of Materials Science and Engineering, Northwestern University, Evanston, Illinois 60208, USA

${ }^{3}$ Department of Physics, Temple University, Philadelphia, Pennsylvania 19122, USA

${ }^{4}$ Advanced Photon Source, Argonne National Laboratory, Argonne, Illinois 60439, USA

${ }^{5}$ Swiss Light Source, Paul Scherrer Institute, CH-5232 Villigen, Switzerland

${ }^{6}$ Advanced Light Source, Lawrence Berkeley National Laboratory, Berkeley, California 94720, USA

${ }^{7}$ Temple Materials Institute, Temple University, Philadelphia, Pennsylvania 19122, USA 
Table S1. The $c$-axis parameters and strain state of as-grown $\mathrm{SrMnO}_{2.5}$ films on LAO, LSAT, and STO, along with the $c$-axis parameters and F content of each film after fluorination.

\begin{tabular}{lllll}
\hline \hline & Substrate & LAO & LSAT & STO \\
& in-plane $(\AA)$ & 3.791 & 3.868 & 3.905 \\
\hline $\mathrm{SrMnO}_{2.5}$ & $c$-axis $(\AA)$ & 3.866 & 3.787 & 3.768 \\
& $\varepsilon(\%)$ & -0.52 & 1.50 & 2.47 \\
$\mathrm{SrMnO}_{2.5-\delta} \mathrm{F}_{\gamma}$ & $c$-axis $(\AA)$ & 3.988 & 3.806 & 3.799 \\
& F content $(\%)$ & $0.22 \pm 0.020$ & $0.24 \pm 0.016$ & $0.31 \pm 0.040$ \\
\hline \hline
\end{tabular}




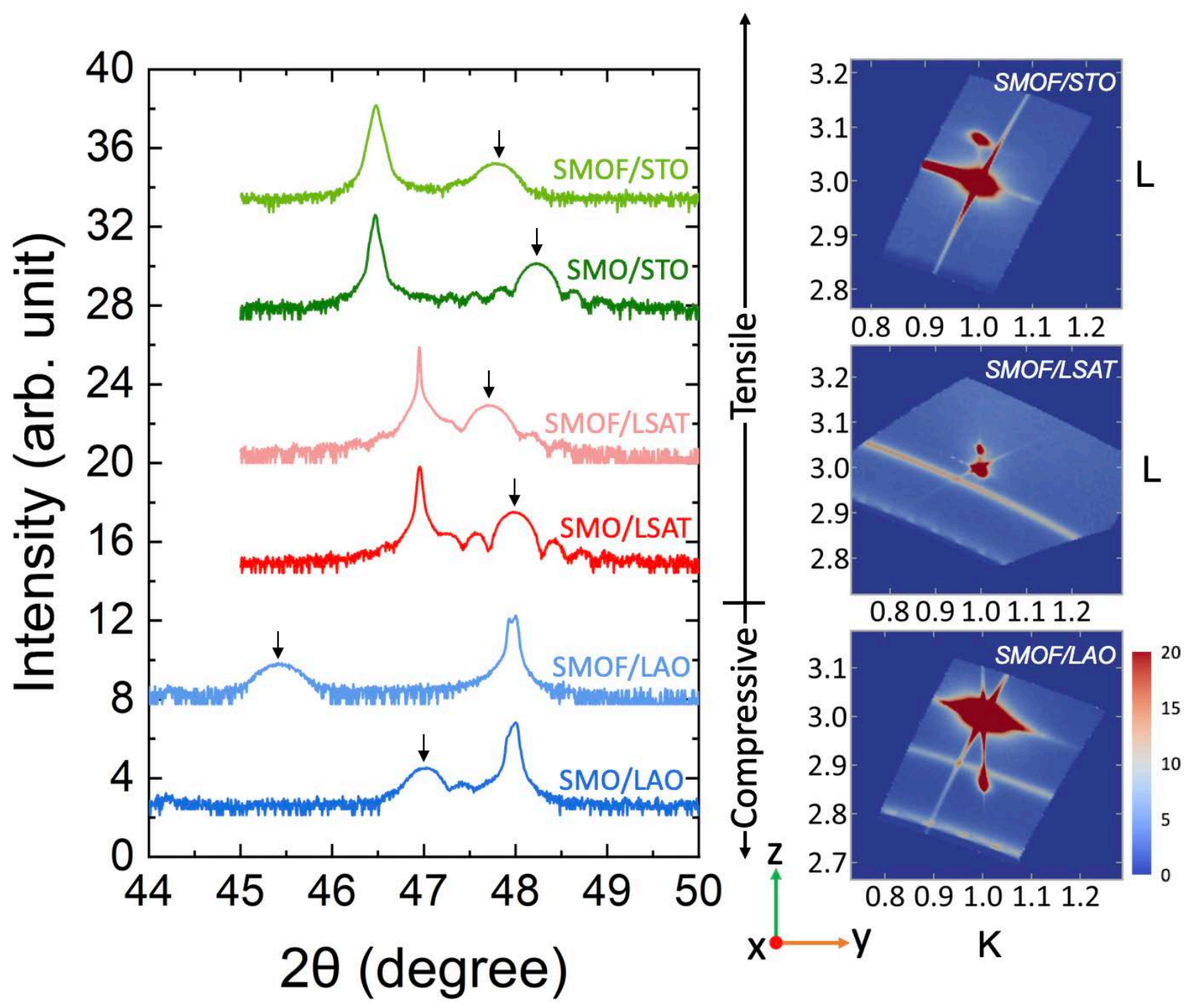

Figure S1. XRD data of as-grown $\mathrm{SrMnO}_{2.5}$ and fluorinated $\mathrm{SrMnO}_{2.5-\delta} \mathrm{F}_{\gamma}$ grown on LAO, LSAT, and STO substrates (left panel). The arrows indicate the 002 film peaks. The RSMs of SMOF on LAO, LSAT, and STO substrates are shown in the right panels. The 113 film peaks of the films appear at the same $K$ position of the substrate, confirming that the films are coherently strained to the substrates. 
(a)

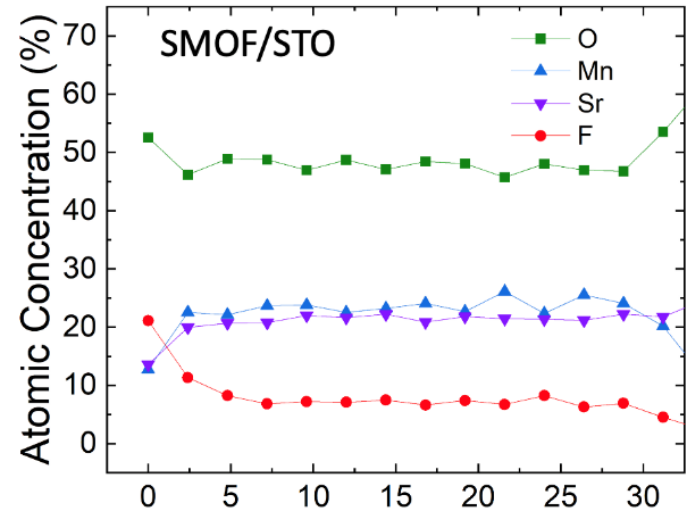

(c)

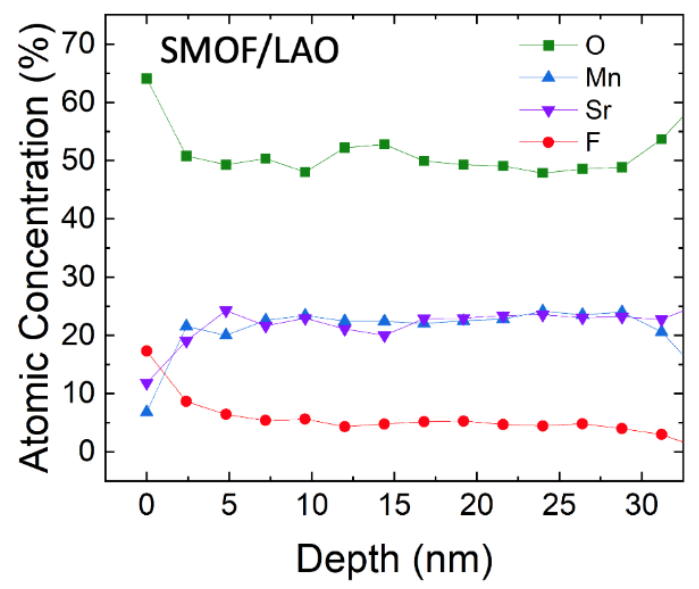

(b)

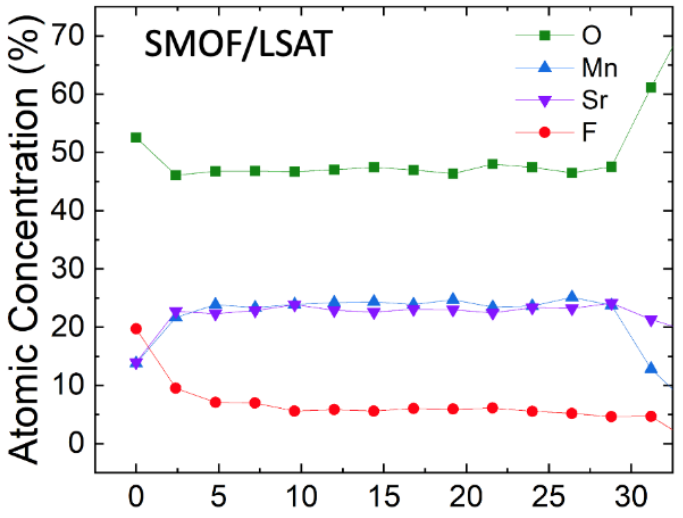

(d)

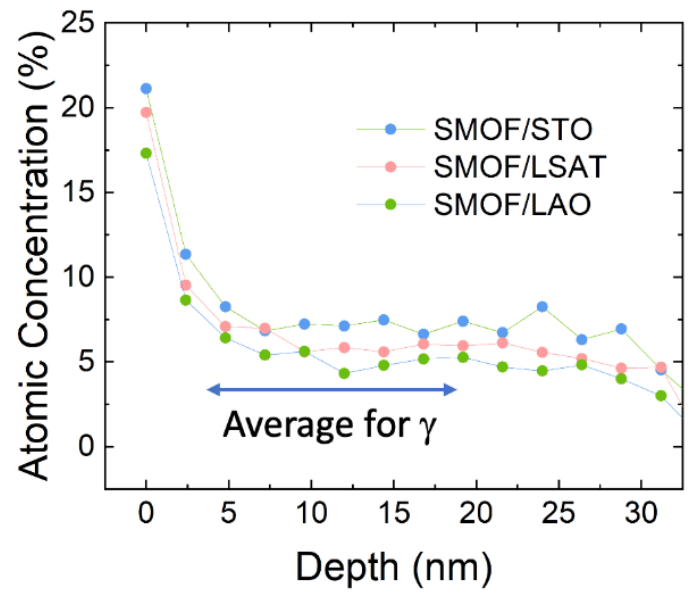

Figure S2. XPS depth profiles showing the atomic concentrations at different sputtering depths of $\mathrm{SrMnO}_{2.5-\delta} \mathrm{F}_{\gamma}$ films grown on (a) STO, (b) LSAT and (c) LAO. The F depth profiles are plotted together in (d). The F concentrations presented in Figure 1(d) were obtained from these same samples. 


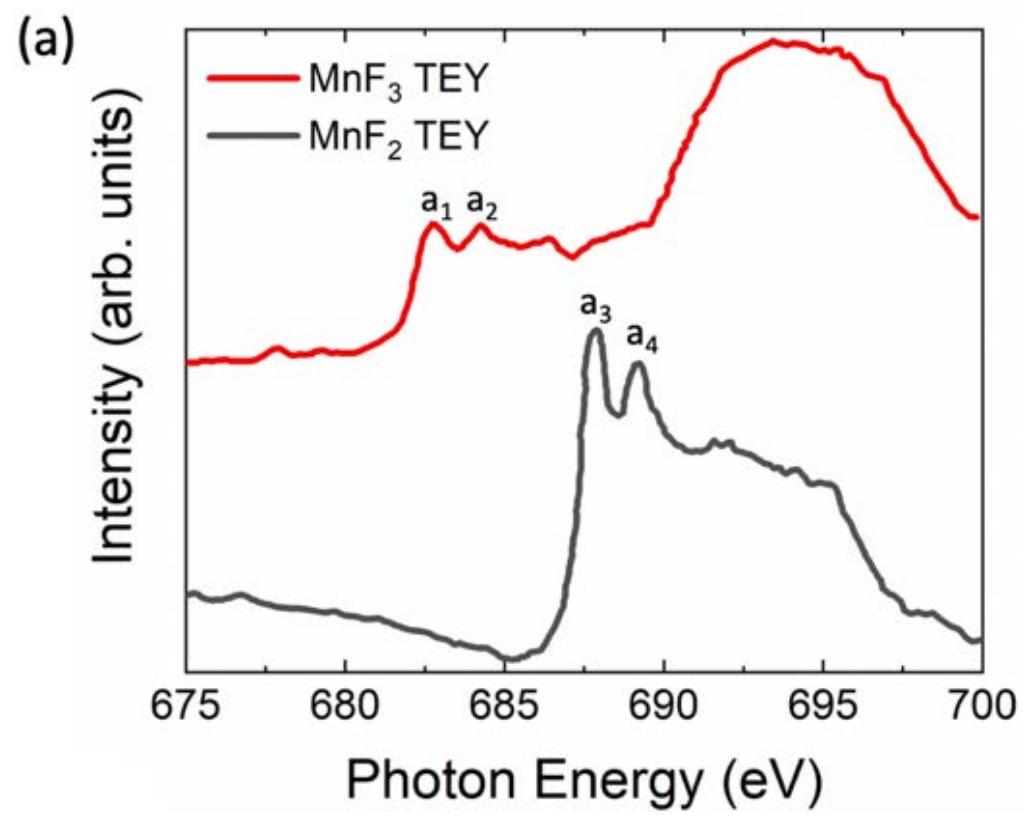

Figure S3. The F K-edge XA spectra of bulk $\mathrm{MnF}_{3}$ and $\mathrm{MnF}_{2}$ as reported by Qiao et al. Data in the figure is reproduced from R. Qiao, T. Chin, S. J. Harris, S. Yan, W. Yang, Curr. Appl. Phys. 13, 544 (2013). 


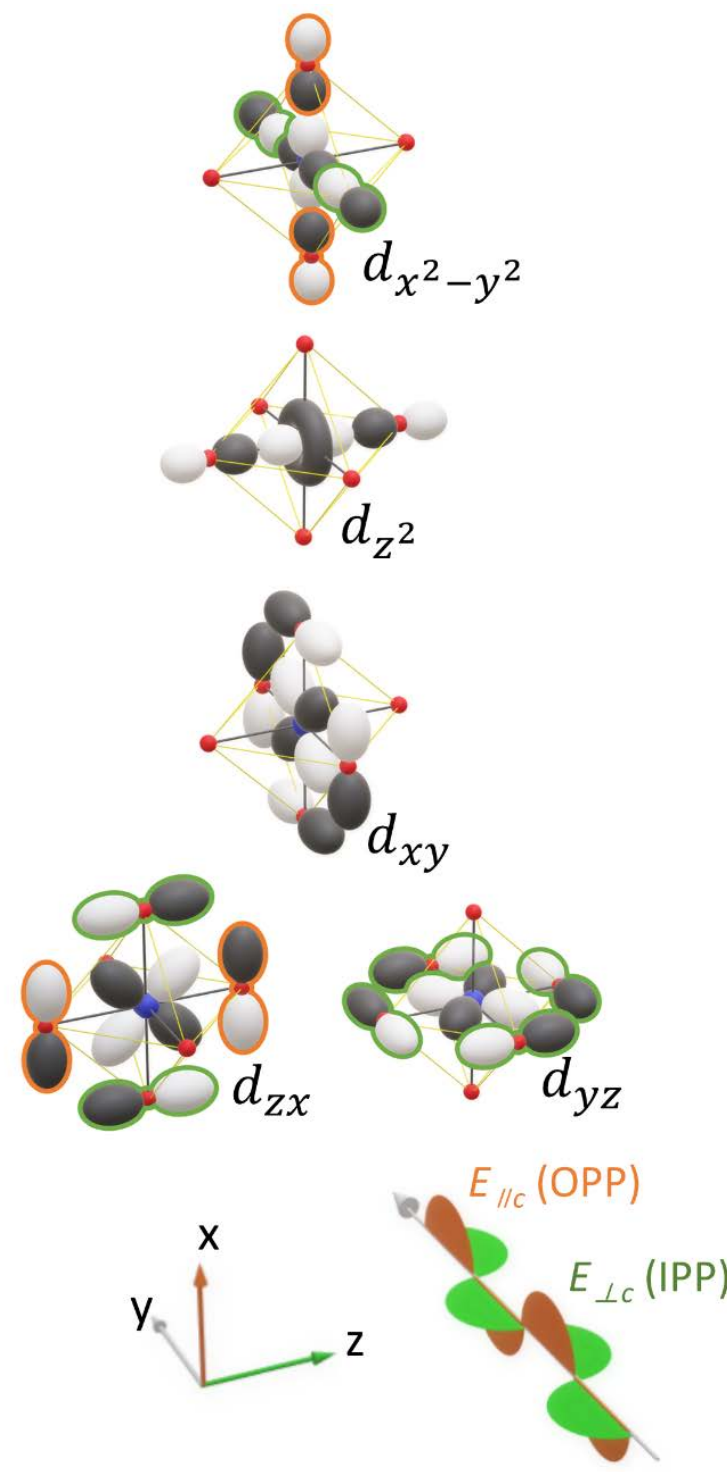

Figure S4. A schematic of $2 p-3 d$ hybridized orbitals as related to the linear polarization of the Xrays. The orbitals are presented in vertical order of their energetic position as determined from DFT calculations. 
(a)

(b)

(c)
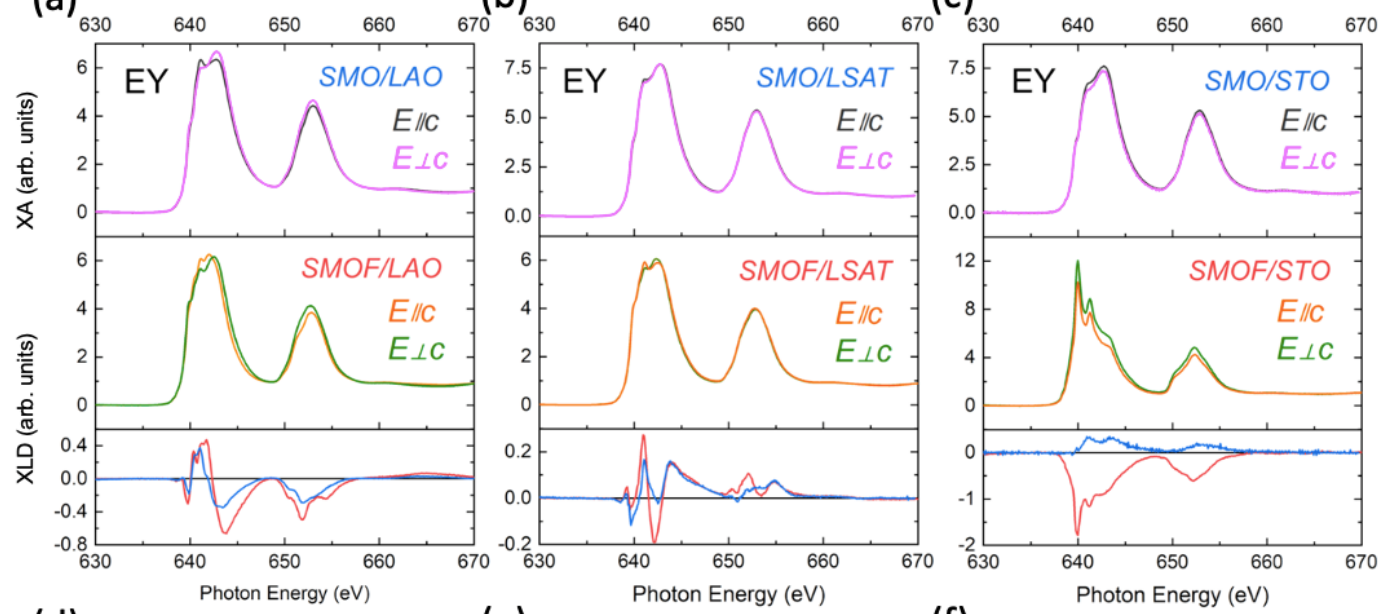

(d)

(e)
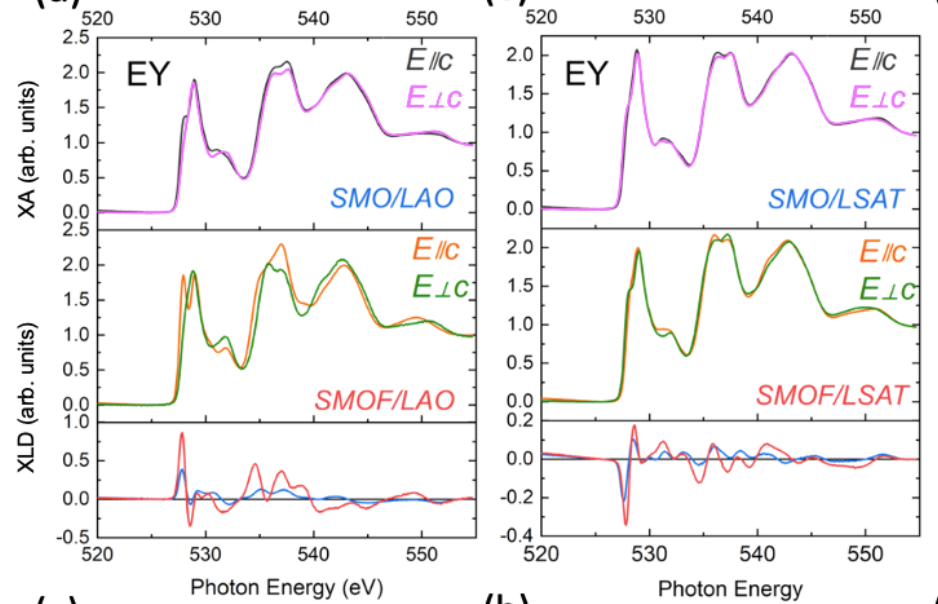

(f)

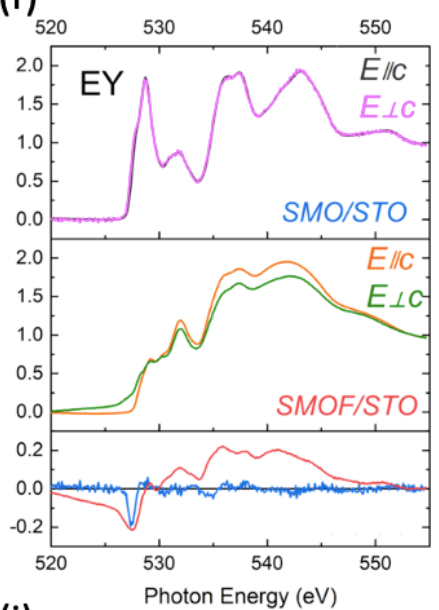

(g)

(h)

(i)
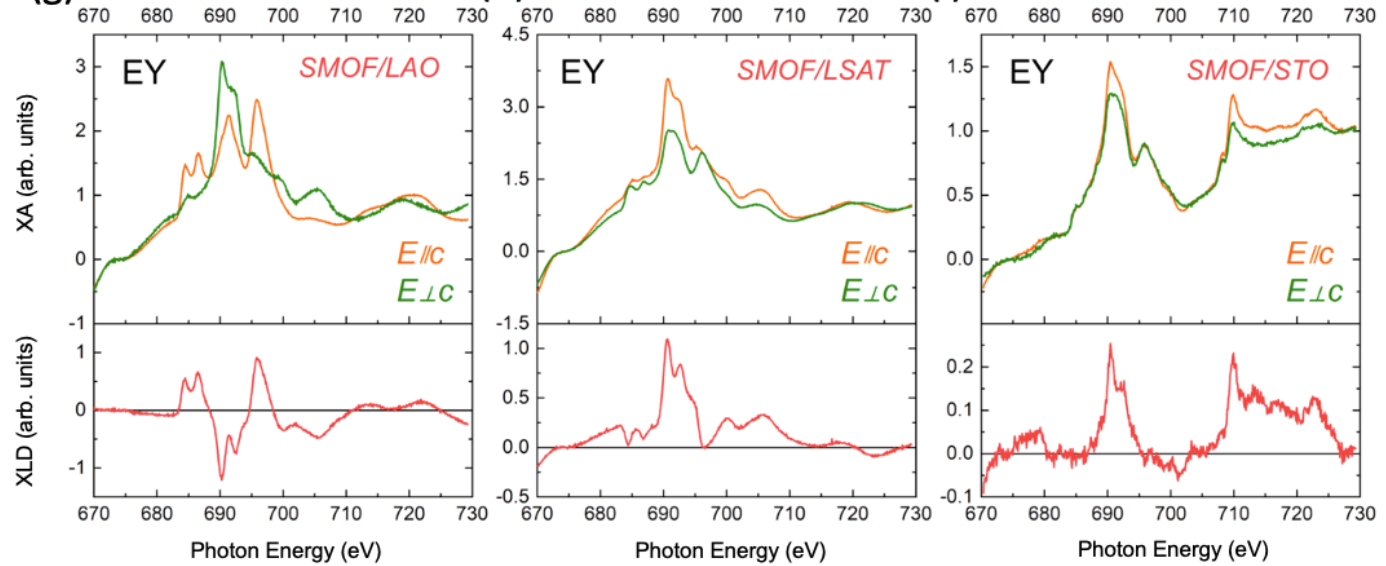

Figure S5. The total electron yield signal obtained at the (a-c) Mn L-edge, (d-f) O K-edge, and (gi) F K-edge of SMO and SMOF films on different substrates. In the bottom panels of (a-f), the blue curve is the linear dichroism from the as-grown SMO and the red curve is the linear dichroism from the SMOF. 

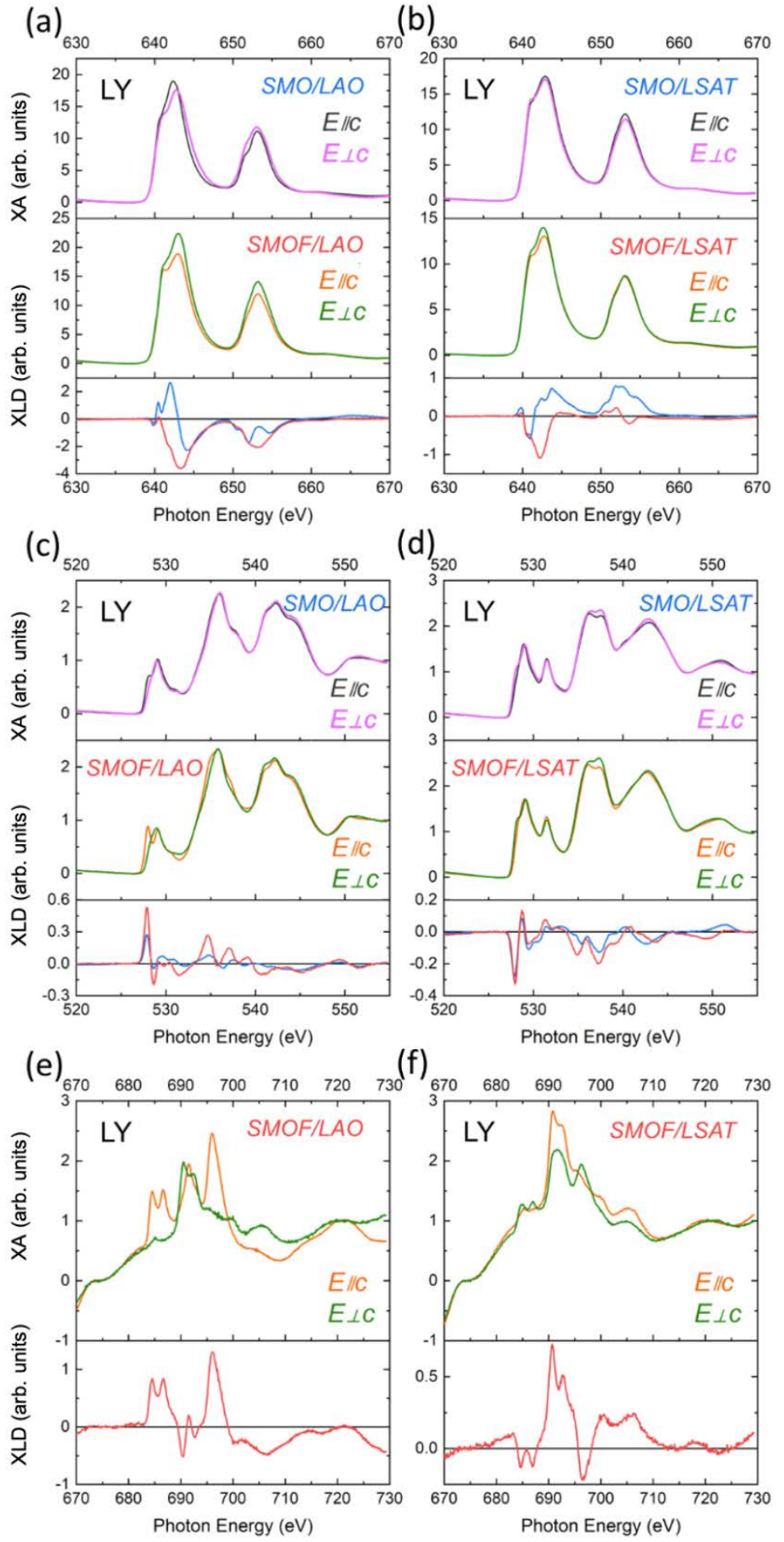

Figure S6. The luminescence yield signal obtained at the (a,b) Mn L-edge, (c,d) O K-edge, and (e,f) F K-edge of SMO and SMOF films. In the bottom panels of (a-d), the blue curve is the linear dichroism from the as-grown SMO and the red curve is the linear dichroism from the SMOF. 

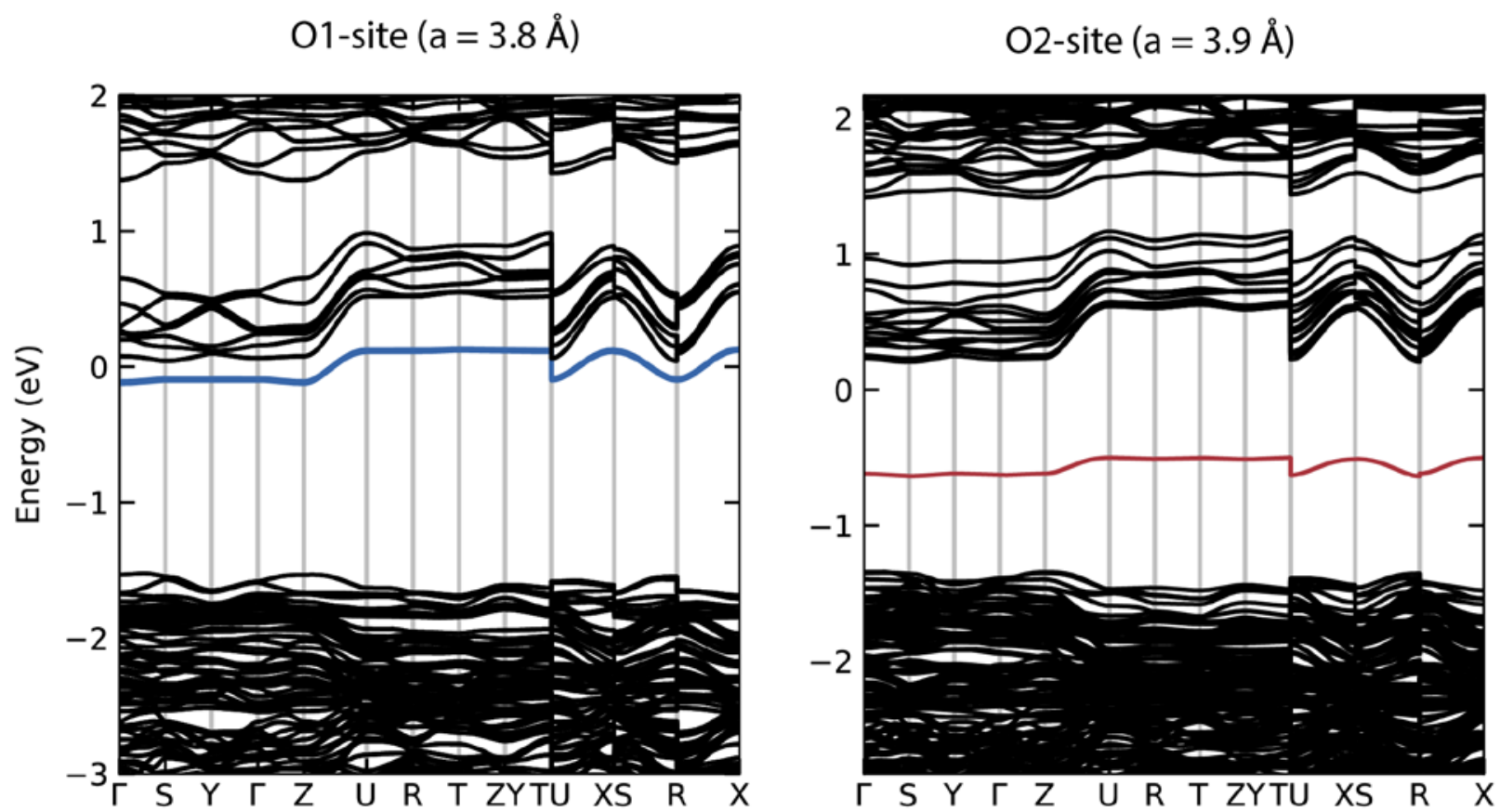

Figure S7. The DFT $+U$ electronic band structures for compressively strained $\mathrm{SrMnO}_{2.5-\delta} \mathrm{F}_{\gamma}$ with $\mathrm{F}$ on the O1-site and tensile strained $\mathrm{SrMnO}_{2.5-\delta} \mathrm{F}_{\gamma}$ with $\mathrm{F}$ on the O2-site. 
(a) $\Gamma \rightarrow X$ reciprocal path

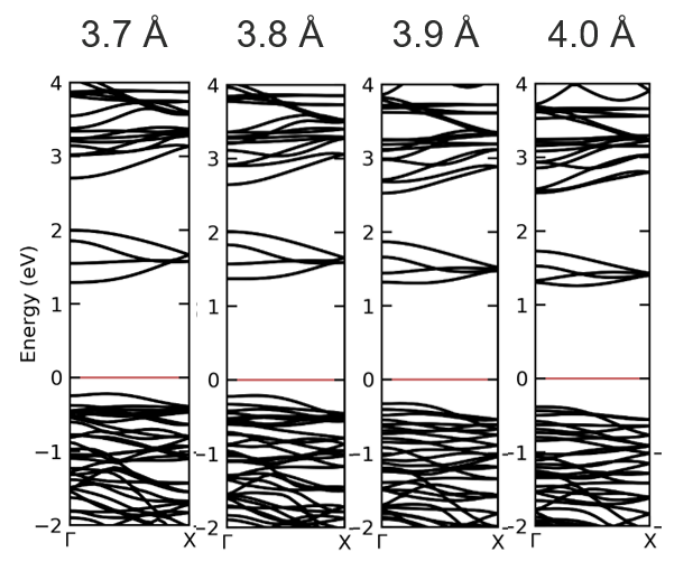

(b) $\Gamma \rightarrow Y$ reciprocal path

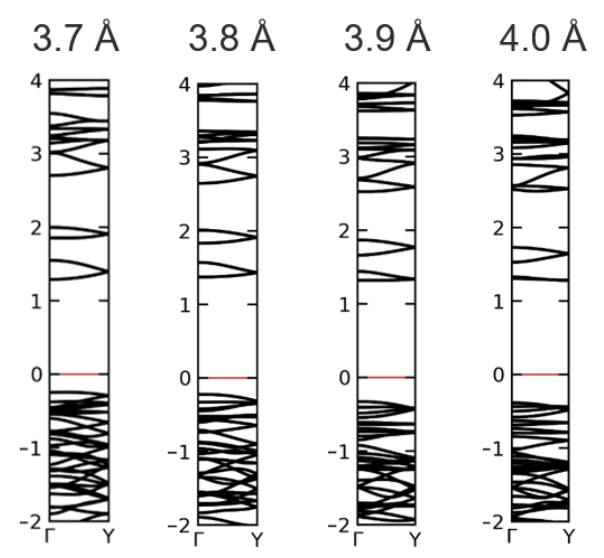

(c) $\Gamma \rightarrow Z$ reciprocal path

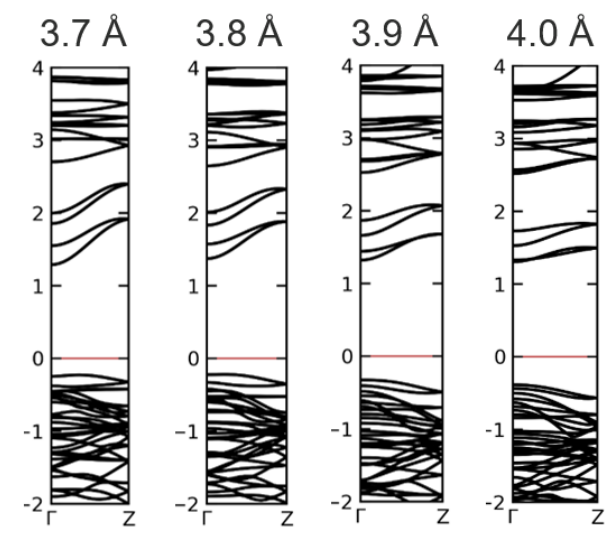

Figure S8. The DFT+U electronic band structures of $\mathrm{SrMnO}_{2.5}$ with different pseudocubic lattice parameters specifying the biaxial constraints along (a) $\Gamma \rightarrow \mathrm{X}$, (b) $\Gamma \rightarrow \mathrm{Y}$, and (c) $\Gamma \rightarrow \mathrm{Z}$. Bandwidth narrowing occurs going from compressive (left panels) to tensile strain (right panels). 
Competing magnetic orders in oxyfluorides film. The E-type and E'-type antiferromagnetic (AFM) orders exhibit zigzag spin order on the $a b$-plane. The two orders differ in the nature of the interplanar magnetic coupling as depicted in Figure S9. The two orders are close in energy in $\mathrm{SrMnO}_{2.5}$ and $\mathrm{SrMnO}_{2.5-\gamma} \mathrm{F}_{\gamma}$ systems. As shown in Table $\mathrm{S} 2, \mathrm{SrMnO}_{2.5}$ exhibits E-AFM as its ground state magnetic order with $\Delta \mathrm{E}_{\mathrm{E}^{\prime}-\mathrm{E}}$ less than $15 \mathrm{meV} / \mathrm{f}$.u, while tensile strain can stabilize E'-AFM order. For the fluorinated compound, $\Delta \mathrm{E}_{\mathrm{E}^{\prime}-\mathrm{E}}$ values are smaller except for $\Delta \mathrm{E}_{\mathrm{E}^{\prime}-\mathrm{E}}$ at 3.7 Å. We adopted E'-AFM order for the discussion in the main text, given that E'-AFM is more stable for the fluorinated material. We note that the biaxial strain dependencies of the E-AFM order are similar (Figure S10): (i) O1-site substitution becomes more stable under compressive strain, and (ii) the $c$-lattice parameter expansion is enhanced with O1-site substitution.

(a) E-type AFM order

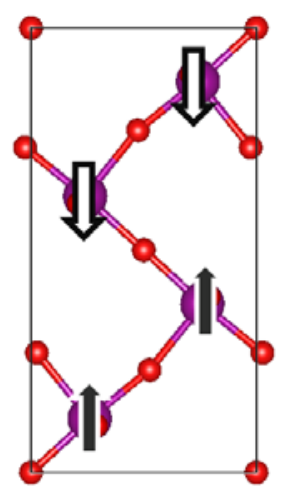

Layer 1

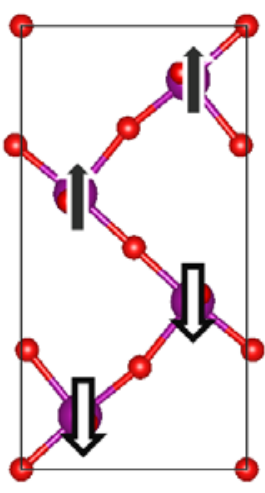

Layer 2 (b) E'-type AFM order

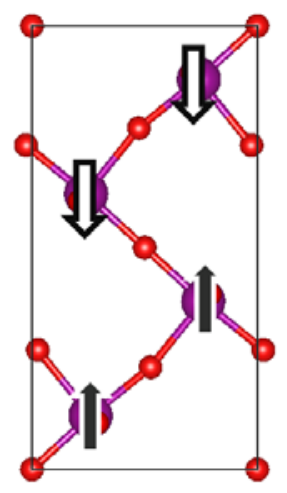

Layer 1

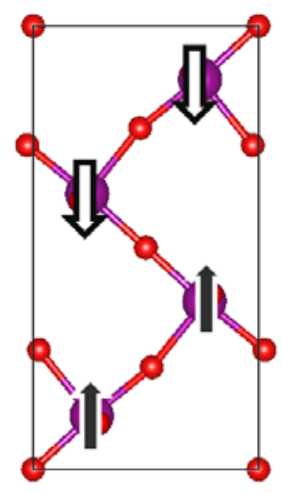

Layer 2

Figure S9. Illustration of two magnetic orders in $\mathrm{SrMnO}_{2.5}$ structure: (a) E-type and (b) E'-type antiferromagnetic order. 
Table S2. Relative energy differences in meV/f.u. between E-AFM and E'-AFM orders $\left(\Delta \mathrm{E}_{\mathrm{E}^{\prime}-\mathrm{E}}\right)$ of $\mathrm{SrMnO}_{2.5}$ and $\mathrm{SrMnO}_{2.5-\gamma} \mathrm{F}_{\gamma}$ (with O2-site substitution) with respect to the in-plane pseudocubic lattice parameter. Positive value means more stable E-AFM order over E'-AFM and vice versa.

\begin{tabular}{lllll}
\hline \hline & $3.7 \AA$ & $3.8 \AA$ & $3.9 \AA$ & $4.0 \AA$ \\
\hline $\mathrm{SrMnO}_{2.5}$ & 7.36 & 14.91 & 11.76 & -3.60 \\
$\mathrm{SrMnO}_{2.5-\gamma} \mathrm{F}_{\gamma}$ & -22.17 & -7.49 & -0.51 & -2.68 \\
\hline \hline
\end{tabular}

(a)

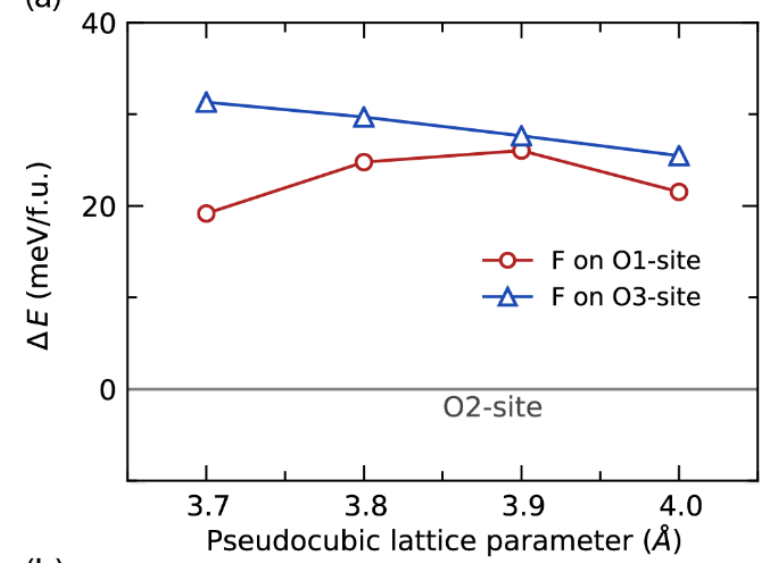

(b)

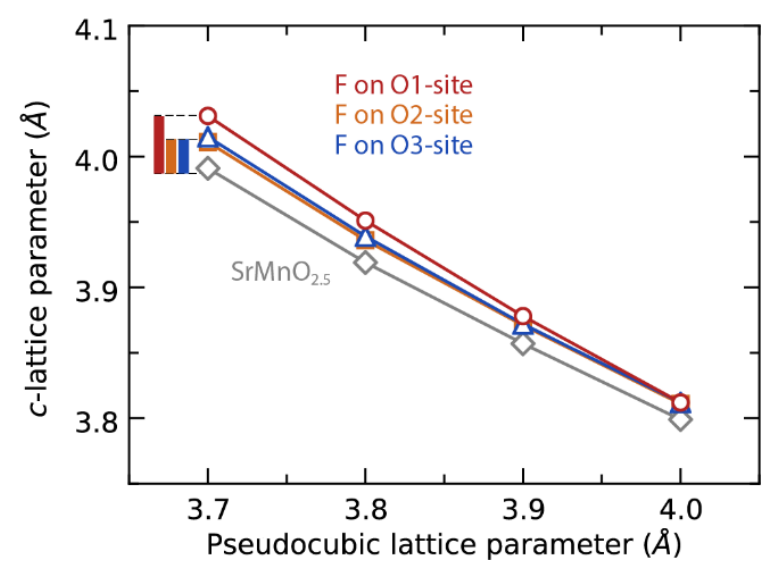

Figure S10. The DFT calculated (a) relative energies and (b) c-lattice parameters of different F occupancies as a function of the in-plane pseudocubic lattice parameter with E-type magnetic order. In panel (a), the energy of the O2-site substitution is set as the reference state. 